\title{
Correction to: Measured PET Data Characterization with the Negative Binomial Distribution Model
}

\author{
Maria Filomena Santarelli ${ }^{1,2} \cdot$ Vincenzo Positano $^{2} \cdot$ Luigi Landini $^{1,2,3}$
}

Published online: 9 February 2018

(c) Taiwanese Society of Biomedical Engineering 2018

\section{Correction to: J. Med. Biol. Eng. (2017) 37:299-312 https://doi.org/10.1007/s40846-017-0236-2}

The article "Measured PET Data Characterization with the Negative Binomial Distribution Model", written by Maria Filomena Santarelli, Vincenzo Positano, Luigi Landini was originally published Online First without open access. After publication in volume [37], issue [3], page [299-312] the author decided to opt for Open Choice and to make the article an open access publication. Therefore, the copyright of the article has been changed to $($ C The Author(s) [2018] and the article is forthwith distributed under the terms of the Creative Commons Attribution 4.0 International License (http://creativecommons.org/licenses/by/4.0/), which permits use, duplication, adaptation, distribution and reproduction in any medium or format, as long as you give appropriate credit to the original author(s) and the source, provide a link to the Creative Commons license, and indicate if changes were made.

The original article can be found online at https://doi.org/10.1007/ s40846-017-0236-2.

Maria Filomena Santarelli

santarel@ifc.cnr.it

1 Institute of Clinical Physiology, National Research Council, via Moruzzi 1, 56124 Pisa, Italy

2 Fondazione CNR-Regione Toscana “G. Monasterio", via Moruzzi, 1, 56124 Pisa, Italy

3 Department of Information Engineering, University of Pisa, via G. Caruso, 16, 56122 Pisa, Italy 